\title{
Aerosol-generating Procedures (AGP): Protective and Partition Measures
}

Airborne or fine droplet spread may be important in transmission of COVID-19 viruses. ${ }^{1}$ The microdroplets $(>5-10 \mu \mathrm{m})$ containing infectious particles may remain suspended in the air. It may be dispersed by air currents, and may be inhaled by a person who is in the same room as the source patient during the AGP. Negative pressure ventilation room and frequent air exchanges can reduce the aerosol but availability of negative pressure room or isolated rooms is scarce. Apart from the airway for anesthesia, bronchoscopy, tracheotomy, ear, nose, throat, and sinus procedures may be regarded as AGP which is illustrated in the following table (Table 1). ${ }^{1}$

During this COVID-19 pandemic, induction of anesthesia and emergence time has never been as challenging time as before. Perhaps the anesthesiologist will be using all measures to prevent and avoid coughing during this time. One of the other challenges is availability of adequate PPE. ${ }^{2}$ It is better to follow WHO or ISA rationale. ${ }^{2,3}$ Some of the other guidelines reported during SARS also may be useful., ${ }^{4,5}$ Absence of negative pressure room, lockdown (closed door) post-AGP may be strictly followed for 20 min to minimize the droplets in air. Hence protocols and management guidelines are necessary, which may differ for each institute, based on availability and innovative equipment. Appropriate education among the caregivers will help to reduce fear and any confusion.

Table 1: List of anesthetic and surgical procedures that are considered aerosol generating ${ }^{7}$

\section{A. List of Surgical Procedures}

- Rigid bronchoscopy

- $\quad$ During the procedure, the airway is not protected and the glottis is stented open by the bronchoscope. Hence effective cough may not be possible. It is likely that forceful expiration may be encountered when a patient is allowed to breathe spontaneously. In some of the patients requiring neuromuscular paralyzing agents and jet ventilation, it is possible that aerosolized particles will be generated. During intermittent ventilation, the use of high flow $\mathrm{O}_{2} .6 \mathrm{~L} / \mathrm{min}$ is highly likely to be required.

- Tracheostomy

- This procedure necessitates circuit disconnection and reconnection. During this procedure maintenance phase circuit leaks, endotracheal cuff or tracheostomy cuff leaks, or malpositioning of a tracheostomy tube are all common and all have the capacity to generate aerosolized droplet. At times, re-intubation may be required if tracheostomy becomes unsuccessful.

- Surgery involving high-speed drilling

- High-speed devices used in dental surgery and orthopedic surgeries were shown to be able to generate an aerosol cloud which could contaminate the theater environment.

\section{B. List of Anesthetic Procedures}

- $\quad$ Airway procedures like awake fiberoptic intubation can produce aerosol droplets which is many a time unavoidable. While preparing airway by topicalization is another incident of producing the large aerosol droplets.

- $\quad$ During mask ventilation

- It has been observed during mask ventilation, tiny droplets are produced and dispersed. It is identified as a risk factor in the spread of infections across healthcare workers. It is likely that more dispersion occurs in those individuals with less experience with mask ventilation.

- Techniques of intubation and extubation

- In patients who are not fully paralyzed during the intubation procedure, aerosol generation is a big risk. During rapid-sequence induction, mask ventilation may still be required to maintain oxygen saturation if difficulty with intubation is encountered. During the procedure of extubation, coughing may occur which can generate aerosols particles. At times, we require suctioning of upper airway, lower airway and high-flow oxygen to maintain oxygen saturation which in turn may also generate aerosols particles.

- Use of high-flow nasal cannula

- Using high-flow nasal cannula is controversial. It requires $40-60 \mathrm{~L} / \mathrm{min}$ of oxygen flow. If nasal prongs are not properly applied, aerosol generation could be enormous.

- Use of noninvasive ventilation

- $\quad$ Experiments using tight-fitted masks with inspiratory positive airway pressure (IPAP) have shown that tiny droplets may still be dispersed from leaks. If we use high IPAP, droplets can disperse to a longer distance.

- $\quad$ Procedure of sputum suctioning

- $\quad$ The coughing is associated with the droplet dispersion. Suctioning may induce coughing and subsequently has the potential to generate aerosolized particles.

- $\quad$ Process of cardiopulmonary resuscitation (CPR)

- $\quad$ During CPR the infection can spread across healthcare workers, as it often involves mask ventilation, airway suctioning, and intubation. The place of CPR may not facilitate control of secretions or aerosolized particle. 


\section{Important Role of a Hospital and the Department of Anesthesiology}

It requires intense efforts from hospital administration authorities and frontline healthcare workers across all disciplines to successfully treat the affected patients. The following measures are recommended for caring suspected and confirmed cases:

- We should develop a diagnosis, management and precaution protocol and workflow guidelines

- We should facilitate triage and prompt diagnosis

- We must design and develop clear workflow guidelines which will help to facilitate multidisciplinary communication among the managing team, medical team, infection control team, and intensive care unit to implement isolation practices.

- We should plan system integration testing of workflow guidelines using medical simulation which in turn helps to ensure robustness.

\section{Training/Education Program}

- Information on disease transmission and prevention to be given to all theater staff to encourage adherence to infection control protocols, possibly via hospital information system, posters and online tutorials.

- We should organize training on proper donning and doffing of personal protective equipment (PPE) including N-95 respirator, goggles, face shield, gowns and gloves.

- Better to familiarize theater staff with the location of gown up (usually outside isolation room) and gown down PPE protocols to avoid cross-contamination.

- We should conduct simulation scenarios involving anaesthesiologists, anaesthetic nurses or assistants to familiarize with modifications in workflow, particularly on induction, extubation, airway crises and cardiopulmonary resuscitation.

- It is good to design cognitive aids to facilitate consolidation of information.

We need to attain airway safely with the least amount of aerosol exposure to the healthcare personnel (Fig. 1). This involves anesthetic team's preparation time without rush and having an area designated for donning and doffing of PPE. Preparation involves
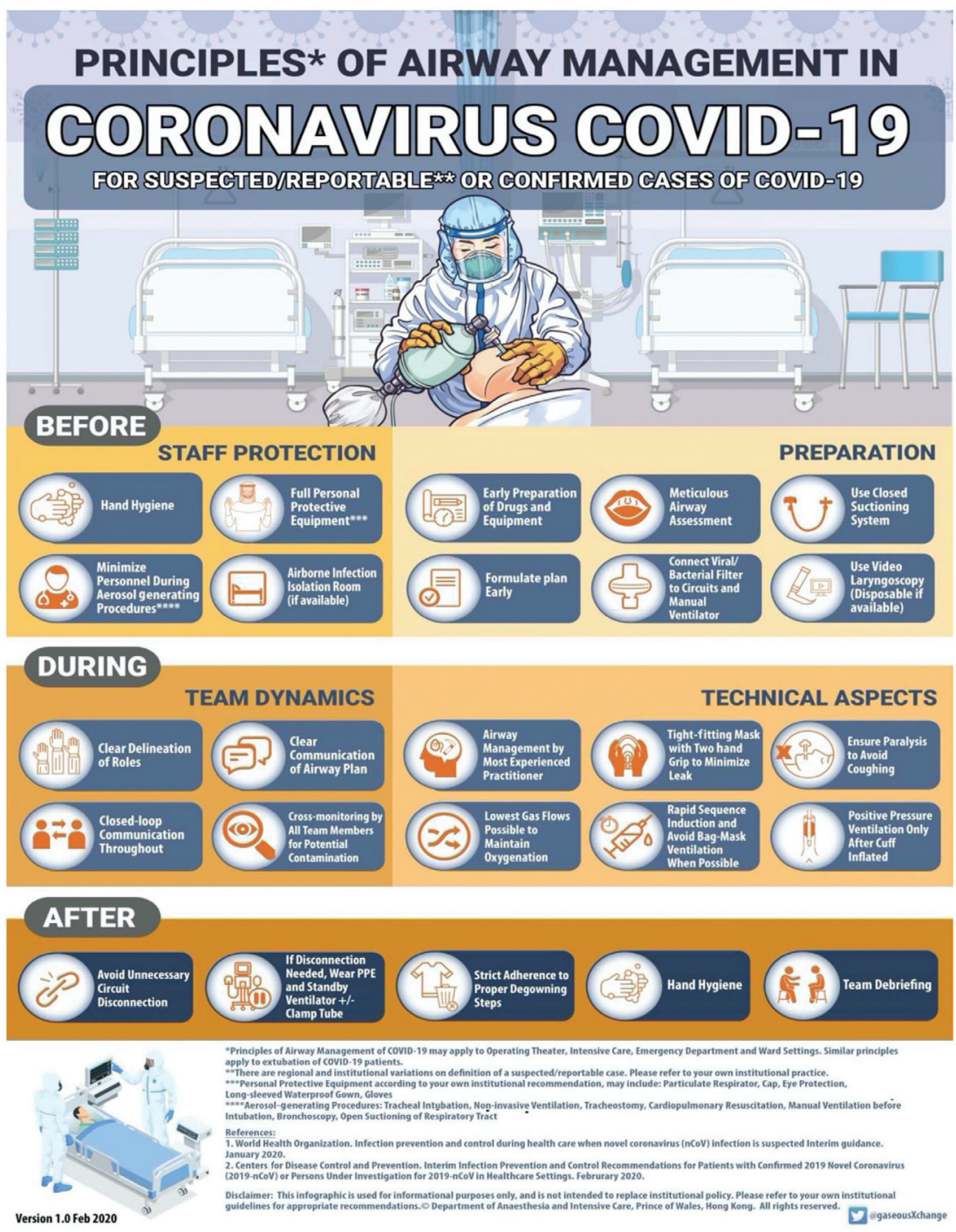

Fig. 1: Principles of safe airway management in suspected/or reportable/or confirmed covid-19 cases $^{6,7}$ 
keeping induction drugs, airway equipment needed for intubation, suction, HME filter and disposable plastic drape ready. Healthcare staff preparing to enter the OR (Operation Room) or any room for airway intervention on a patient with suspected or confirmed 2019$\mathrm{nCoV}$, should be prepared to use the N-95 mask, double gloves, fluid-resistant gown, head cover and the face shield to minimize skin exposure to droplet contamination. After donning comes the actual intubation process, then extubation, and lastly, doffing off the PPE.

Consider premedication with glycopyrrolate or atropine to reduce secretions. It is preferable to adapt rapid sequence induction by an experienced anesthetist who is good at intubation. It is ideal to have an anesthetic nurse or technician for help. Avoid mask ventilation, once intubated cuff is inflated. Positive pressure ventilation should be commenced only after intubated cuff is inflated. It is good to insert bougie or stylet with the endotracheal tube (ETT) to avoid repeated attempts of laryngoscopy. There should be minimal staff in the OR but two anesthetists are preferable (even if it's a trainee) along with a nurse or a technician. Second pair of hands will be helpful in giving best clear view of larynx through plastic drapes by adjustment. They will help in using other equipment like a video laryngoscope for intubation. They will also be helpful in connecting ETT to the circuit as quickly as possible, securing airway and communicate with other teams outside the OR by noncontact method.

Patient always arrives with a mask on into the OR while this plastic partition is being prepared. A clear plastic sheet is used as a protective barrier between the patient and the procedure performing person during AGP5. It may limit the spread of potential coughrelated viral load or blast of expired air beyond the inner surface of the drape. It is attached by tape to the supportive rods on the operating table near the chest and head end. (Figs 2 and 3 show the view through the plastic sheet). Partition remains in place after the

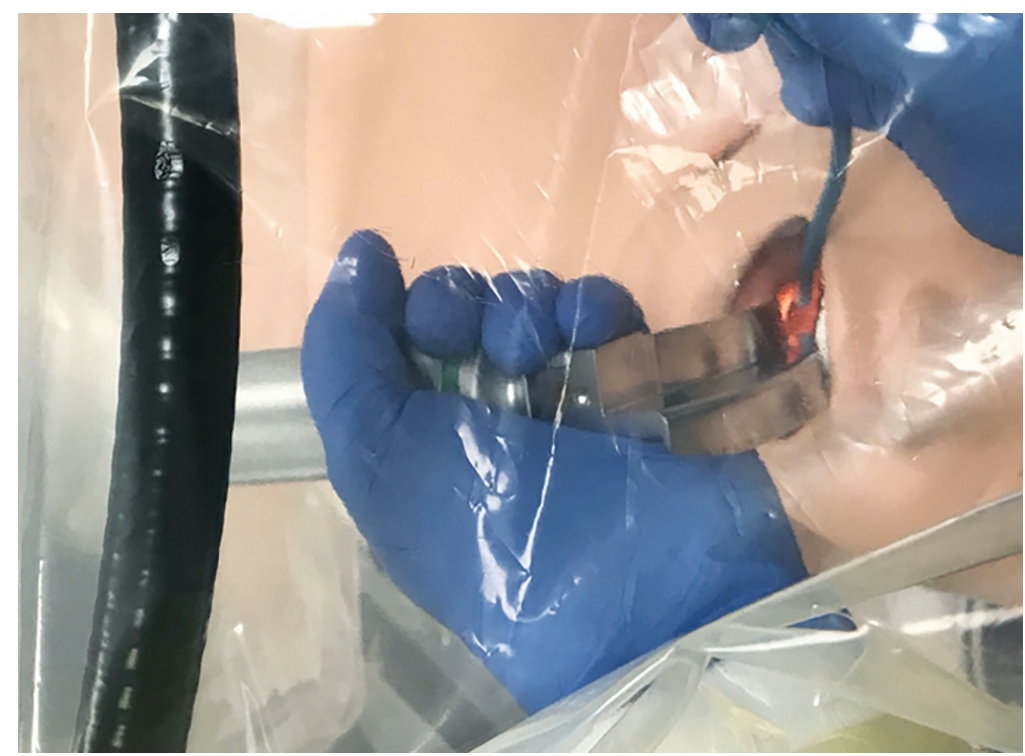

Fig. 2: Photo showing use of black surgical screen holders and transparent plastic drape to form a protective partition. Note the anesthesiologist's hands are beneath the drape with bougie in laryngeal inlet to facilitate rail-roading ETT

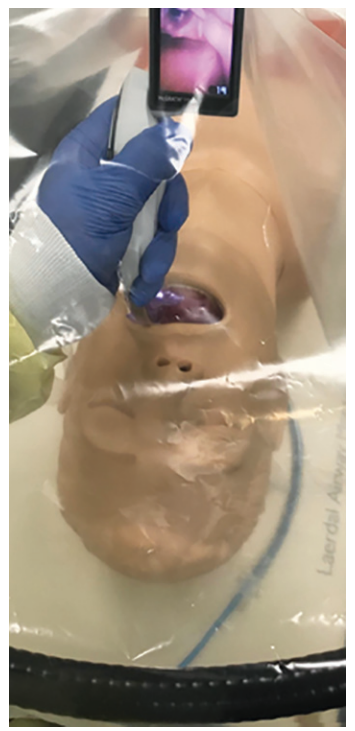

Fig 3: Photo showing use of black surgical screen holders and transparent plastic drape to form a protective partition 
extubation until the patient recovers and ready to be shifted to a ward with the mask on. Then plastic partition should be disposed of along with the other items.

Pre-oxygenation should be done for five minutes with $100 \% \mathrm{O}_{2}$ followed by RSI (rapid sequence induction). Ensure hydrophobic filter placed between mask and breathing circuit. Once intubation is confirmed by end-tidal $\mathrm{CO}_{2}$, mechanical ventilation is commenced. Any contaminated airway should be disposed of by the technician or sent for decontamination, and the team should wait for 20 min. During any part of the procedure, it's very important to avoid circuit disconnection. After 20 min, surgeons may enter the OR to perform the procedure. Same anesthesia team who had performed the intubation, should be available in the room during the extubation time. After extubation observe lockdown for another 20 minutes and then the OR may be cleaned. All staff leaves the OR to doff off the PPE in a systematic manner. Dispose PPE only after the patient is stable with respiratory parameters in normal range and the team is satisfied with airway management. Avoid touching your hand to any part of your body and perform hand hygiene very often. Doffing off is carefully done: remove gloves from dominant hand to the base of a thumb, and then using dominant hand remove the gloves of non-dominant hand. Perform hand hygiene, avoid touching central part of the gown (most contaminated), undo the knot and lean forward to pull the gown away. Roll the gown touching inner aspect of it and dispose it. Next remove eye protection wear while leaning forward, and then remove mask and perform hand hygiene. All contaminated materials including plastic sheet to be disposed in designated, biomedical waste bins.

To conclude, during COVID-19 pandemic, anesthetic induction and emergence has never been as challenging time as before. COVID-19 is a highly contagious disease, posing a huge burden on the healthcare system. It is our duty to provide optimal care to patients and protect healthcare workers and other patients from spread of this disease. Goal is to avoid placing the face of the intubating person close to the patient. This partition equipment along with the PPE which has been illustrated here may help to minimize the droplet spread during the AGP.

Vasanth Rao Kadam

Associate Professor, University of Adelaide, South Australia, 5011, Australia drvasanthrao@yahoo.com

\section{References}

1. Modes of transmission of virus causing COVID-19: implications for IPC precaution recommendations. 27th March 2020. Scientific brief from the World Health Organization.

2. World Health Organization's Rational Use of Personal Protective Equipment for Coronavirus Disease-2019. WHO, Interim guidance, Accessed March 29, 2020.

3. Malhotra N, Joshi M, Datta R, Bajwa SJ, Mehdiratta L. Indian Society of Anaesthesiologists (ISA National). Advisory and position statement regarding COVID-19. Indian J Anaesth 2020;64:259-63

4. Kamming D, Gardam M, Chung F. Anaesthesia and SARS. Br J Anaesth. 2003;90(6):715-718.

5. Matava CT, Yu J, Denning S. Clear plastic drapes may be effective at limiting aerosolization and droplet spray during extubation: implications for COVID-19. Can J Anaesth. 2020 Apr 3. doi: 10.1007/s12630-020-01649.

6. World Health Organization infection prevention and control during health care when novel coronavirus infection is suspected. Interim guidance Jan 2020.

7. Dr. Grace Tang, Dr. Albert Kam Ming Chan. Perioperative management of suspected/confirmed cases of COVID-19 (6 April 2020) ATOTW 421 —www. wfsahq.org/resources/anaesthesia-tutorial-of the-week. 